\title{
Comparison Study of Resource Allocation Strategies for OFDM Multimedia Networks
}

\author{
Cédric Guéguen ${ }^{1}$ and Sébastien Baey ${ }^{2}$ \\ ${ }^{1}$ IRISA/Université de Rennes 1, Campus de Beaulieu, 35042 Rennes cedex, France \\ ${ }^{2}$ LIP6/CNRS, UPMC Sorbonne Universités, 4 place Jussieu, 75005 Paris, France
}

Correspondence should be addressed to Cédric Guéguen, cedric.gueguen@irisa.fr

Received 23 February 2012; Revised 11 June 2012; Accepted 12 June 2012

Academic Editor: Yi Su

Copyright ( 92012 C. Guéguen and S. Baey. This is an open access article distributed under the Creative Commons Attribution License, which permits unrestricted use, distribution, and reproduction in any medium, provided the original work is properly cited.

Advanced MAC scheduling schemes provide efficient support of multimedia services in multiuser OFDM wireless networks. Designed in a cross layer approach, they opportunistically consider the channel state and are well adapted to the wireless multipath fading environment. These schedulers take advantage of time, frequency, and multiuser diversity. Thereby they maximize the global system throughput while ensuring the highest possible level of fairness. However their performances heavily depend on the bandwidth granularity (i.e., the number of elementary resource units) that is used in the resource allocation process. This paper presents and compares the main OFDM scheduling techniques. In particular it studies the influence of bandwidth granularity on the resource allocation strategies performances. The paper reveals that though bandwidth granularity has never been considered in former studies, it is of major importance for determining the application range of advanced OFDM scheduling techniques.

\section{Introduction}

Bandwidth allocation in next generation broadband wireless networks is a challenging issue. The schedulers shall provide mobile multimedia transmission services with an adequate Quality of Service (QoS). These new multimedia services with tight QoS constraints require increased system capacity together with fairness.

The past decades have witnessed intense research efforts on wireless communications. In contrast with wired communications, wireless transmissions are subject to many channel impairments such as path loss, shadowing, and multipath fading [1-4]. These phenomena severely affect the transmission capabilities and in turn the QoS experienced by applications, in terms of data integrity but also in terms of the supplementary delays or packet losses which appear when the effective bit rate at the physical layer is low. Among all candidate transmission techniques for broadband transmission, Orthogonal Frequency Division Multiplexing (OFDM) has emerged as the most promising physical layer technique for its capacity to efficiently reduce the harmful effects of multipath fading. This technique is already widely implemented in most recent wireless systems like $802.11 \mathrm{a} / \mathrm{g}$ or 802.16. The basic principle of OFDM for fighting the effects of multipath propagation is to subdivide the available channel bandwidth in subfrequency bands of width inferior to the coherence bandwidth of the channel (inverse of the delay spread). The transmission of a high speed signal on a broadband frequency selective channel is then substituted with the transmission on multiple subcarriers of slow speed signals which are very resistant to intersymbol interference and subject to flat fading. This subdivision of the overall bandwidth in multiple channels provides frequency diversity. Added to time and multiuser diversity, this may result in a very spectrally efficient system subject to an adequate scheduling.

More recently intense research efforts have been given in order to propose efficient schedulers for OFDM-based wireless networks. Opportunistic schedulers, in particular, have received much attention [5-9]. These schemes preferably allocate the resources to the active mobile(s) with the most favourable channel conditions at a given time instant. This takes maximal profit of the multiuser diversity and frequency diversity in order to maximize the system throughput. 
In fact, these schedulers highly rely on diversity for offering their good performances. Several studies analyze precisely the influence of the multiuser diversity on the performances of the reference schedulers [10-16]. However no work is focused on the role of frequency diversity which highly relies on bandwidth granularity. In this paper, we consider a fixed amount of bandwidth. Following the OFDM principle, this bandwidth is divided into subfrequency bands called subcarriers. In the allocation process, the different subcarriers are grouped for constituting the elementary resource units that are allocated to the mobiles. The higher the number of subcarriers in a group, the less the freedom and the (bandwidth) granularity in the allocation process and, consequently, the less the frequency diversity and the efficiency of the opportunistic resource allocation process. It is interesting to note that it is however not always interesting to maximize the bandwidth granularity. Indeed, the higher the bandwidth granularity is the more efficient are these schedulers, but it is at the expense of supplementary signaling overhead that can become prohibitive. A tradeoff has to be found.

In this paper, we study the influence of bandwidth granularity on the performances of the OFDM reference schedulers considering multiple metrics: system throughput, delay, jitter, fairness, and so forth. In contrast with multiuser diversity, the effect on the performances of bandwidth granularity is much more complex to analyze. Some papers compare the most acknowledged opportunistic schedulers but often show conflicting results. We show in this paper that this is mainly due to differences in bandwidth granularity hypotheses while each reference scheduler has its specificities and is built on specific assumptions. And, in particular, the performances of these schedulers strongly depend on the bandwidth granularity since, for example, some are primarily designed for single carrier communications, some for multicarrier systems. This paper clearly shows how a given scheduler can outperform another depending on the bandwidth granularity available in the system and why. We also provide in this paper the application range of each of the main OFDM reference schedulers with respect to bandwidth granularity.

The outline of the paper is as follows. Section 2 provides an overview of the OFDM system and a definition of bandwidth granularity. The main OFDM scheduling algorithms are described in the next section. Section 4 gives a detailed evaluation of the performance of these reference schedulers in a large range of bandwidth granularity values. Section 5 discusses the application range of each of the schedulers based on the results of Section 4 . Section 6 concludes the paper.

\section{System Description and Frequency Granularity}

We consider Orthogonal Frequency Division Multiple Access (OFDMA). The physical layer is operated in TDD mode using the frame structure described in Figure 1 which ensures a good compatibility with existing systems like the OFDM-based transmission mode of the IEEE 802.16-2004
$[17,18]$. The total available bandwidth is divided into subfrequency bands or subcarriers. The subcarrier spacing is constant and equal to a value inferior to the coherence bandwidth of the channel. Following [19], it is chosen in the order of $15 \mathrm{kHz}$. The total number of subcarriers is denoted $n_{\text {sub }}$. These subcarriers are grouped in $n_{g}$ groups,

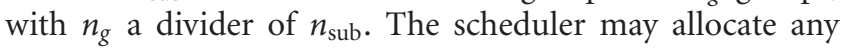
of these groups to any mobile. Consequently the higher the number $n_{g}$ of groups, the higher the amount of bandwidth granularity and the higher the offered frequency diversity in the bandwidth allocation process.

The radio resource is further divided in the time domain in frames. Each frame is itself divided in time slots of constant duration. The time slot duration is an integer multiple of the OFDM symbol duration. Moreover, the frame duration is fixed to a value much smaller than the coherence time (inverse of the Doppler spread) of the channel. With these assumptions, the transmission on each subcarrier is subject to flat fading with a channel state that can be considered static during each frame. Transmissions performed on different subcarriers by different mobiles have independent channel state variations [20]. On each subcarrier, the modulation scheme is QAM with a modulation order adapted to the channel state between the access point and the mobile to which it is allocated. This provides the flexible resource allocation framework required for opportunistic scheduling. Note that the higher the bandwidth granularity $n_{g}$, the more flexible the allocation and the higher the diversity. The elementary resource unit (RU) is defined as any (group of subcarrier, time slot) pair. Each of these RUs may be allocated to any mobile.

\section{Overview of the OFDM Reference Schedulers}

We consider a centralized and synchronized approach [21]. The packets originating from the backhaul network are buffered in the access point which schedules the downlink transmissions (Figure 2). In the uplink, the mobiles signal their traffic backlog to the access point which builds the uplink resource mapping.

The MAC protocols currently used in wireless networks were originally and primarily designed for wired networks. These classical access methods like Round Robin (RR) and Random Access (RA) are not well adapted to the wireless environment and provide poor throughput. Consequently, much interest has recently been given to the design of scheduling schemes that maximize the performance of multiuser OFDM systems. In the following, we focus on the three major scheduling techniques that emerged: Maximum Signal-to-Noise Ratio (MaxSNR), Proportional Fair (PF), and Weighted Fair Opportunistic (WFO) scheduling.

3.1. Maximum Signal-to-Noise Ratio Scheduling. Many schemes are derived from the Maximum Signal-to-Noise Ratio (MaxSNR) technique (also known as Maximum Carrier-to-Interference ratio (Max C/I)) which allocates the resource at a given time to the active mobile with the greatest SNR $[5,6,22]$. Denoting $m_{k, n}$ the maximum number of bits that can be transmitted on a time slot of Resource Unit $n$ if 


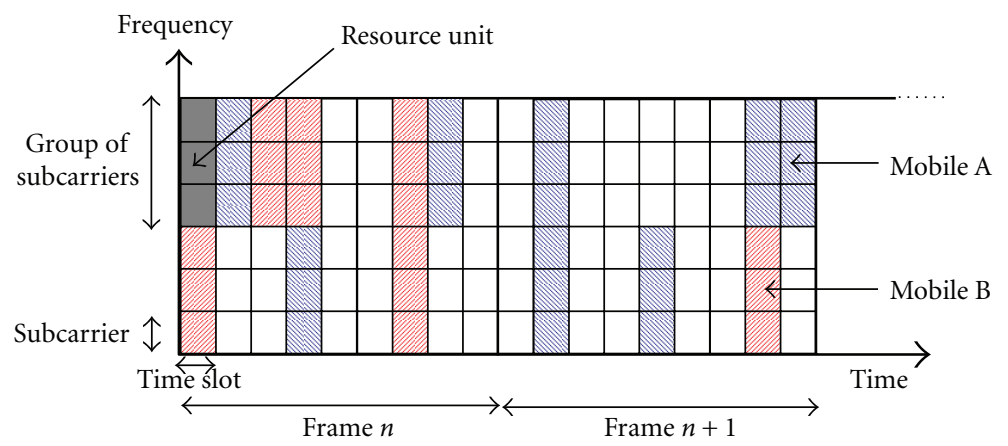

Figure 1: Frame structure in TDD mode $\left(n_{\text {sub }}=6\right.$ and $\left.n_{g}=2\right)$.

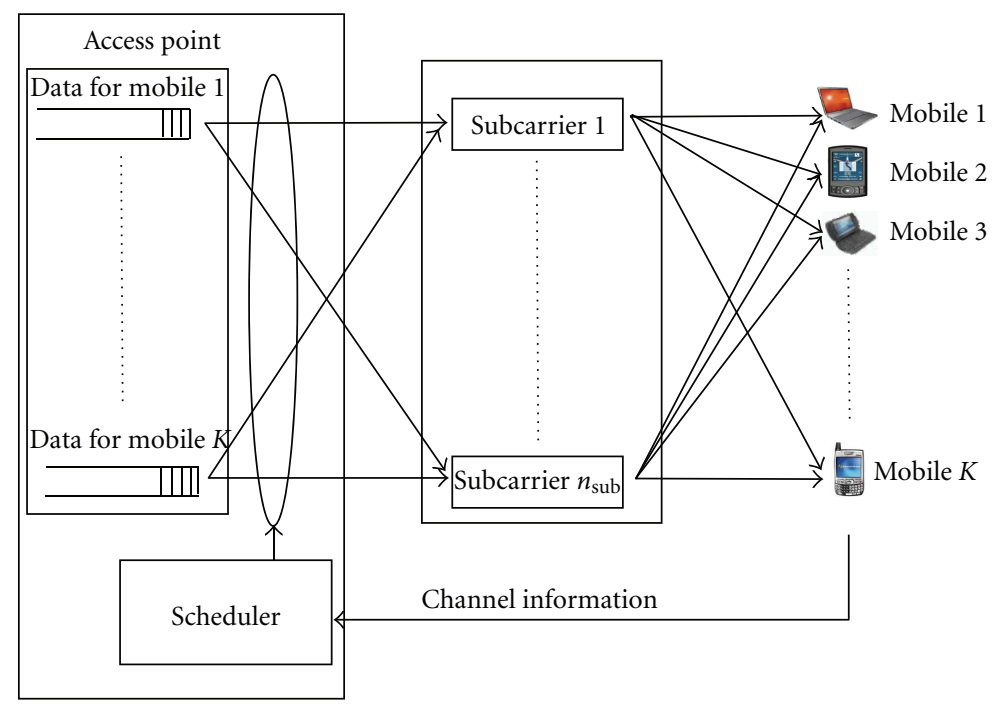

FIGURE 2: Opportunistic allocation of the radio resources among the set of mobiles in the downlink.

it is allocated to the mobile $k$, MaxSNR scheduling consists in allocating the RU $n$ to the mobile $j$ which has the greatest $m_{k, n}$ such as

$$
j=\underset{k}{\operatorname{argmax}}\left(m_{k, n}\right), \quad k=1, \ldots, K,
$$

where $K$ is the total number of mobiles.

Profiting of multiuser and bandwidth granularity, MaxSNR continuously allocates the radio resources to the mobile with the best spectral efficiency. Consequently, MaxSNR strongly increases the system throughput. Dynamically adapting the modulation and coding allows to always make the most efficient use of the radio resource and come closer to the Shannon limit. However MaxSNR assumes that the user with the most favourable transmission conditions has information to transmit at the considered time instant. It does not take into account the variability of the traffic and the queuing aspects. Additionally, a negative side effect of this strategy is that the closest mobiles to the access point have disproportionate priorities over mobiles more distant since their path loss attenuation is much smaller. This results in a severe lack of fairness.
3.2. Proportional Fair Scheduling. Proportional Fair (PF) algorithms have recently been proposed to incorporate a certain level of fairness while keeping the benefits of multiuser diversity $[7,8,23-26]$. The basic principle is to allocate resources to a mobile $j$ when its channel conditions are the most favourable with respect to its time average such as

$$
j=\underset{k}{\operatorname{argmax}}\left(\frac{m_{k, n}}{M_{k, n}}\right), \quad k=1, \ldots, K,
$$

where $M_{k, n}$ is the time average of the $m_{k, n}$ values.

At a short time scale, path loss variations are negligible and channel state variations are mainly due to multipath fading, statistically similar for all mobiles. Thus, PF provides an equal sharing of the total available bandwidth among the mobiles as RR. Applying an opportunistic scheduling approach, the system throughput increase is comparable to the MaxSNR gain [9]. Actually, PF combines the advantages of the classical schemes and the opportunistic schemes. It currently appears as the best bandwidth management scheme. 
In PF-based schemes, fairness consists in guaranteeing an equal share of the total available bandwidth to each mobile, whatever its position or channel conditions. However, since the farther mobiles have a lower spectral efficiency than the closer ones due to pathloss, all mobiles do not all benefit of an equal average throughput despite they all obtain an equal share of bandwidth. This induces heterogeneous delays and unequal QoS. [12, 23, 27] demonstrate that fairness issues persist in PF-based protocols when mobiles have unequal spatial positioning, different traffic types, or different QoS targets. In particular PF scheduling does not take into account the delay constraints and is not well adapted to multimedia services which introduce heterogeneous users, new traffic patterns with highly variable bit rates and stringent QoS requirements in terms of delay and packet loss.

3.3. Weighted Fair Opportunistic Scheduling. More recently a new MAC scheduler, called "Weighted Fair Opportunistic (WFO)," has been proposed for efficient support of multimedia services in multiuser OFDM wireless networks $[9,28]$. Built in a higher layers/MAC/PHY cross-layer approach, this scheme is designed for best profiting of the multiuser diversity and taking advantage of the dynamics of the multiplexed traffics. It takes into account both the transmission conditions and the higher layer constraints (traffic patterns, QoS constraints). In order to provide an efficient support of multimedia transmission services, WFO dynamically favors the mobiles that go through a critical period with respect to their QoS requirements using dynamic priorities.

Evaluating if a mobile goes through a critical period should not only focus on the classical mean delay and jitter analysis. Indeed, a meaningful constraint regarding delay is the limitation of the occurrences of large values. Accordingly, [9] defines the concept of delay outage by analogy with the concept of outage used in system coverage planning. A mobile $k$ is in delay outage (in critical period) when its packets experience a delay greater than a given threshold $T_{k}$ defined by the mobile application requirements. The delay experienced by each mobile is tracked all along the lifetime of its connection. At each transmission of a packet of mobile $k$, the ratio of the total number of packets whose delay exceeded the threshold divided by the total number of packets transmitted since the beginning of the connection is computed. The result is called Packet Delay Outage Ratio (PDOR) of mobile $k$ and is denoted $\mathrm{PDOR}_{k}$. This measure is representative of the emergency for the mobile $k$ to be served. Figure 3 illustrates an example cumulative distribution of the packet delay of a mobile at a given time instant. A mobile can be considered as satisfied when, at the end of its connection, its delay constraint is met, that is, its experienced PDOR is less than a PDOR target specific to the mobile application.

The WFO scheduling principle is to allocate a Resource Unit $n$ to the mobile $j$ which has the greatest WFO parameter value $\mathrm{WFO}_{k, n}$ with

$$
j=\underset{k}{\operatorname{argmax}}\left(\mathrm{WFO}_{k, n}\right), \quad k=1, \ldots, K,
$$

where $\mathrm{WFO}_{k, n}$ is equal to

$$
\mathrm{WFO}_{k, n}=m_{k, n} \times f\left(\mathrm{PDOR}_{k}\right),
$$

with $f$ a strictly increasing polynomial function defined in [9].

With this scheduling, physical layer information (represented through the factor $m_{k, n}$ ) are used in order to take advantage of the time, frequency and multiuser diversity and maximize the system capacity. Higher layer information (represented through the factor $f\left(\mathrm{PDOR}_{k}\right)$ ) are exploited in order to introduce dynamic priorities between the flows for ensuring the same QoS level to all mobiles. With this original weighted system that introduces dynamic priorities between the flows, WFO keeps a maximum number of flows active across time but with relatively low traffic backlogs. This results in a well-balanced resource allocation. Preserving the multiuser diversity allows to continuously take a maximal benefit of opportunistic scheduling and thus maximize the bandwidth usage efficiency. When the bandwidth granularity is sufficient, WFO better conceals the system capacity maximization, QoS support and fairness objectives than PF and MaxSNR as will be observed in the next section.

\section{Performance Evaluation}

In this section, we study the allocation of radio resources among the set of mobiles situated in the coverage zone of an access point using the classical Round Robin (RR) and the most efficient opportunistic schedulers presented in Section 3: MaxSNR, PF, and WFO. Performance evaluation results are obtained using OPNET discrete event simulations. We focus on five essential performance criteria:

(i) offered system capacity;

(ii) mean buffer occupancy;

(iii) delay and jitter;

(iv) perceived QoS satisfaction level.

4.1. Simulation Setup. In the simulations, $n_{t s}$ is defined as the number of time slots available in a frame. We assume a total number $n_{\text {sub }}$ of 128 subcarriers and a total number $n_{t s}$ of 5 time slots in a frame. In addition, the frame duration is fixed to $2 \mathrm{~ms}$ so that the channel state can be considered static during each frame. The results are analyzed with respect to different bandwidth granularities: $n_{g}$ ranges between 1 and 128 which, respectively, corresponds to groups of 128 subcarriers to 1 subcarrier.

A detailed description of the channel model is given in [28]. The channel gain model on each subcarrier assumes free space path loss and multipath Rayleigh fading [4]. We consider a reference distance $d_{\text {ref }}$ for which the free space attenuation equals $a_{\text {ref. }}$. As a result the channel gain is given by

$$
a_{k, n}=a_{\mathrm{ref}} \times\left(\frac{d_{\mathrm{ref}}}{d_{k}}\right)^{3.5} \times \alpha_{k, n}^{2},
$$


where $d_{k}$ is the distance to the access point of the mobile owning the service flow $k$ and $\alpha_{k, n}^{2}$ represents the flat fading experienced by this service flow $k$ if transmitted on the RU $n$. In the following, $\alpha_{k, n}$ is Rayleigh distributed with an expectancy equal to unity. The maximum transmit power satisfies

$$
10 \log _{10}\left(\frac{P_{\max } T_{s}}{N_{0}} \times a_{\text {ref }}\right)=31 \mathrm{~dB}
$$

Additionally, the BER target is taken equal to $10^{-3}$.

We consider 8 mobiles which run a videoconference application with successive connections of five minutes duration. The traffic is composed of an MPEG-4 video stream [29] multiplexed with an AMR voice stream [30]. This demanding type of application, typical of multimedia, generates a high volume of data with high sporadicity and requires tight delay constraints which substantially complicates the task of the scheduler. The average bit rate of each source is $80 \mathrm{Kbps}$. The PDOR target is set to $5 \%$ and the delay threshold $T_{k}$ is fixed to the value of $80 \mathrm{~ms}$ considering real time constraints.

As described in Section 3, the main performance differences between MaxSNR, PF, and WFO regards fairness with respect to mobiles located at unequal positions. This paper proposes to study the influence of the frequency granularity on the performances of the schedulers. Though never studied before, this factor has a very strong impact on the performances of the schedulers. In order to facilitate the analysis, we have chosen to represent only the results of the simulations that involve static users. Simulations with mobile users have also been carried out but when mobility is considered, the results are much more complex to analyze with respect to bandwidth granularity. The mobility model actually interferes with the performances of the schedulers and the role of the bandwidth granularity then could not clearly be isolated. Consequently, in the presented simulation results and in order to observe how fairness is affected by the amount of bandwidth granularity available in the system, a first half of mobiles are situated close to the access point $(2.5 \mathrm{~km})$ so that their mean $m_{k, n}$ value equals 3 bits per subcarrier and per time slot. The second half of the mobiles are situated twice over farther $(5 \mathrm{~km})$.

4.2. Qualitative Impact of Frequency Granularity on the Scheduling Performances. The performances of the OFDM reference schedulers described in Section 3 depend on the amount of bandwidth granularity available in the system. Each scheduler is more or less sensible to an increase of the bandwidth granularity.

Figure 4 shows the distribution of the number of bits that may be transmitted per subcarrier and per time slot by far and close mobiles, considering the channel model described in [28]. In Figure 4(a), the bandwidth granularity is low: $n_{g}$ equals 1 , that is, the system accommodates only 1 group of 128 subcarriers. Figure 4(b) corresponds to the highest bandwidth granularity: $n_{g}$ equals 128 , that is, there are 128 groups of 1 subcarrier.

Note that the number of bits that may be transmitted on a subcarrier can only be a discrete number. Indeed, in

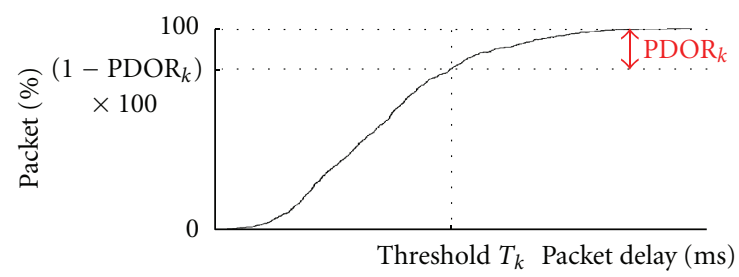

FIgURE 3: Example packet delay CDF and experienced PDOR.

the system under study, the elementary data unit is a bit. Hence, during a time slot, only an integer number of bits can be transmitted on a subcarrier. This explains why when $n_{g}$ equals 128 (128 groups of 1 subcarrier), the distribution of the number of bits that may be transmitted on a subcarrier is constituted only with discrete and integer values (as shown in Figure 4(b)). Similarly, when $n_{g}$ equals 1 , the scheduler has only one capacious Resource Unit (RU) to allocate. This RU is constituted by one group of 128 subcarriers that may be allocated to a single mobile only. As in Figure 4(b), the total number of bits that is then transmitted on the 128 subcarriers during a timeslot remain integer and discrete but are much higher since they relate globally to the 128 subcarriers. In order to compare the results on a same basis, we computed in Figure 4(a) the number of bits that may be transmitted on average per subcarrier when $n_{g}$ equals 1 (and not on a group of subcarriers).

Figure 4(a) shows that the distributions of the number of bits that may be transmitted on a subcarrier for far and for close mobiles when $n_{g}$ equals 1 are very narrow compared to the case where $n_{g}$ equals 128 (Figure 4(b)). Indeed, when the subcarriers are grouped, the quality fluctuations of the subcarriers compensate each other inside the group. As a consequence of this averaging effect, the capacity of a group of subcarriers is pretty much constant which in turn offers less degrees of freedom in the resource allocation at system level. This is why the performances worsen in terms of capacity.

Additionally, we observe in Figure 4(a) that, in contrast with Figure 4(b), the distributions obtained for the two groups of mobiles have no intersection. When all subcarriers are grouped, the mean number of bits that may be transmitted by close mobiles over a group of subcarriers is always larger than the transmit possibility of far mobiles due to the averaging effect. This results in a really unfair allocation for MaxSNR-based schedulers when all subcarriers are grouped since close mobiles then have absolute priority over far mobiles. In contrast, when subcarriers may be allocated individually, close mobiles may get a chance at some time instant to gain access to a subcarrier when its fading conditions are favorable on this subcarrier. This is why the higher the value of $n_{g}$, the lower the unfairness of the MaxSNR-based scheduler.

4.3. System Capacity. We first studied the system capacity offered by the four scheduling algorithms as a function of the bandwidth granularity. Figure 5 analyses the impact of 


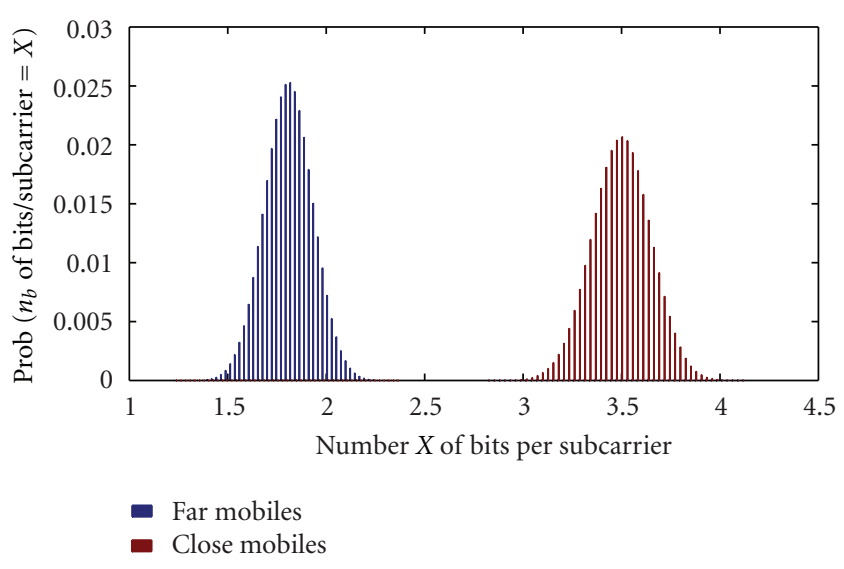

(a) With 1 group of 128 subcarriers $\left(n_{g}=1\right)$

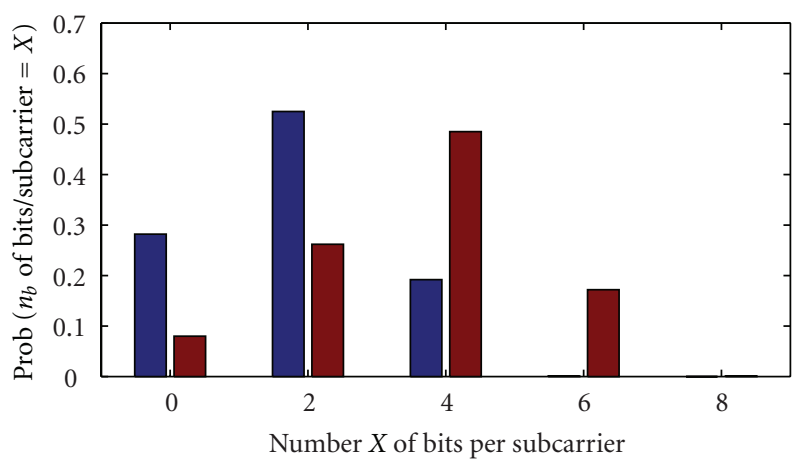

- Far mobiles

- Close mobiles

(b) With 128 groups of 1 subcarrier $\left(n_{g}=128\right)$

FIGURE 4: Number of bits per subcarrier considering two distinct bandwidth granularities.

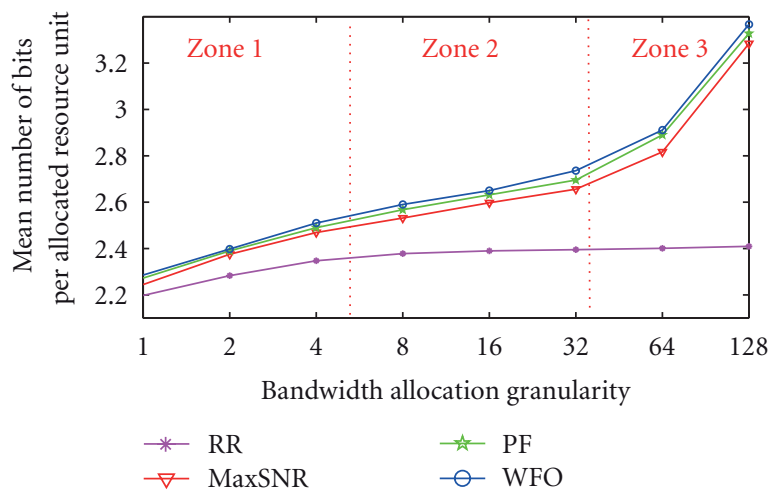

(a) Spectral efficiency per RU

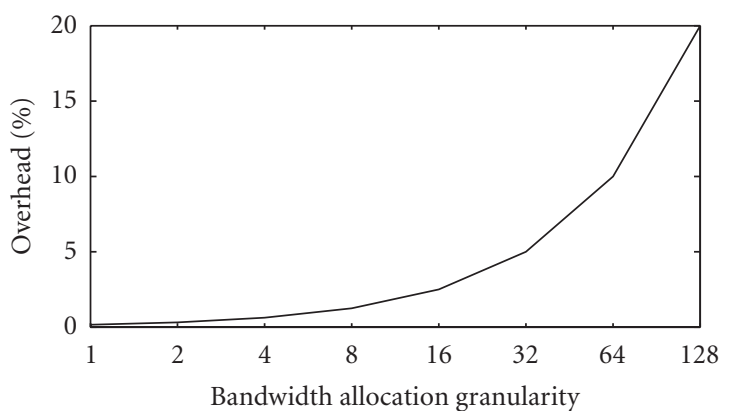

(b) Overhead

FIGURE 5: Impact of bandwidth granularity on spectral efficiency and overhead.

a bandwidth granularity increase on spectral efficiency and overhead.

The first effect is a spectral efficiency increase as shown in Figure 5(a) which gives the average number of bits carried on a time slot of a used subcarrier by each tested scheduler under diverse bandwidth granularities. As expected, RR does not take benefit of multiuser diversity which results in a bad utilization of the bandwidth and, in turn, poor system throughput. Based on opportunistic scheduling, the three other schemes globally show better performances. Improving the spectral efficiency of the system, less RUs are needed than with RR for managing the same amount of traffic, and the system capacity increases.

The system capacity of a wireless network actually highly relies on the level of spectral efficiency that is provided by the scheduler. The purpose of the opportunistic schedulers is to allocate the radio resources at a given instant to the mobiles that have the best radio conditions and have data to transmit at that time instant. If the considered mobile does not have data to transmit, the resource unit is allocated to another mobile whose radio conditions are worse. Consequently the spectral efficiency decreases. Thus, multiuser diversity plays a central role for the maximization of the system capacity. The specificity of the opportunistic schedulers is that they take benefit of the multi-user, time and frequency diversity in order to increase this spectral efficiency and consequently the system capacity.

For each scheduler, we observe an interesting inflection of the curve when the bandwidth granularity increases resulting from the superimposition of the following three phenomena.

(i) The first is predominant mainly in Zone 1 and affects all the schedulers. We observe a spectral efficiency increase which is due to the sparing of many subcarriers used for the transmission of only few bits. To be clearer, let us consider that a given mobile has only a few bits to transmit. If $n_{g}$ equals 1 , the scheduler has only capacious Resource Units to allocate. As a consequence, many subcarriers are allocated (128 subcarriers if $n_{g}$ equals 1 ) while maybe 
one or two could be sufficient. Consequently, the excess of allocated subcarriers in the group is wasted while with more frequency granularity they could be allocated to another mobile. This yields a huge waste of resource. The elementary shares of resource are too large. With a value of $n_{g}$ of 2 , the scheduler allocates 2 groups of 64 subcarriers and this waste is reduced by approximately $50 \%$. With a value of $n_{g}$ of 4 , it is reduced by about $75 \%$, and so forth. Thus, an interesting capacity gain may be obtained with only a little more bandwidth granularity. Sufficient granularity is then provided in the scheduling for allocating an amount of resource that quite closely matches the need of the mobile.

(ii) In conjunction to this, a second phenomenon affects the curves of the opportunistic schedulers only. This phenomenon is clearly visible in Zone 2 and 3 where the curve of the RR is nearly flat as opposed to the curves of the opportunistic schedulers which exhibit a sensible inflection. This shows that they take advantage of the supplementary bandwidth granularity when $n_{g}$ increases. As explained in Section 4.2, when the bandwidth granularity increases, the distribution values of the number of bits that may be transmitted on an RU spreads out. With more available combination choices in the bandwidth allocation, MaxSNR, $\mathrm{PF}$, and WFO increase their system capacity gain.

(iii) The third phenomenon dominates in Zone 3 mainly, accentuating the inflection of the curve of the opportunistic schedulers. When a group of subcarriers is allocated, some subcarriers of this group may go unused because their quality is very poor. They are nevertheless wasted because they belong to the allocated group. This waste totally disappears when the bandwidth granularity is maximal. Indeed, when $n_{g}$ equals 128 , if a subcarrier is not usable by a given mobile, it is spared for another mobile. As a general rule, a higher bandwidth granularity helps to avoid transmitting no bit at all on some subcarriers of a group. This results in a higher spectral efficiency.

The second effect of a bandwidth granularity increase is a greater signaling overhead. Figure 5(b) represents the price to pay in terms of signaling as a function of the bandwidth granularity. Indeed, for each allocated group of subcarriers, the scheduler must signal which mobile is chosen. Consequently, when $n_{g}$ increases, a greater part of the bandwidth is wasted. Indeed, denoting $n_{s}$ the number of (subcarrier, time slot) pairs needed to transmit this information, the overhead ratio can be defined as

$$
\rho=\frac{n_{g} \times n_{s}}{n_{f} \times n_{\text {sub }} \times n_{t s}},
$$

where $n_{f}$ is the number of frames allocated during one scheduling step. We considered that the scheduling process allocates the RUs frame by frame. Consequently $n_{f}$ is set to 1. Additionally, we assume that $n_{s}$ can be set to 1 since the information to signal can be represented by a small integer ( since $n_{\text {sub }}$ is set to 128 and $n_{t s}$ is set to 5 ).
Figure 6 combines the results of Figures 5(a) and 5(b). It presents the bandwidth usage ratio that is defined as the mean number of allocated RUs divided by the total number of RUs in the frame, including both the useful data and the signaling overhead. Considering only the results of Figure 5(a), we could expect that the higher the bandwidth granularity, the greater the spectral efficiency, the lower the bandwidth usage ratio, and the greater the remaining capacity for accommodating other potential users. However, the supplementary signaling overhead cannot be ignored and Figure 6 shows that, for each scheduler, a high bandwidth granularity $n_{g}$ induces too much overhead which can not be compensated by a better frequency diversity use. These results yield the application range of each scheduler. RR's best performances are obtained when $n_{g}$ equals 8 , MaxSNR and PF provide their best performances when $n_{g}$ equals 16 and WFO performances which strongly rely on diversity reach their optimum when $n_{g}$ equals 32 .

The bandwidth usage ratio results have been collected through simulation. However, it is possible to relate the spectral efficiency and overhead ratio to the bandwidth usage ratio as follows. The bandwidth usage ratio is equal to the total average bit rate of data to transmit divided by the system capacity. In our simulation, we use 8 sources with an average bit rate of $80 \mathrm{Kbps}$. This corresponds to a total average bit rate of $640 \mathrm{Kbps}$. Let us denote $n_{\mathrm{bRU}}$ as the mean number of bits per allocated RU (Figure 5(a)), $\rho$ the overhead ratio (Figure $5(\mathrm{~b})$ ), and $n_{f p s}$ the number of frames to allocate per second. The system capacity is

$$
C=\left(n_{\mathrm{sub}} \times n_{t s} \times n_{f p s}\right) \times n_{\mathrm{bRU}} \times(1-\rho) .
$$

For example, with $\mathrm{RR}$, when $n_{g}$ equals 1 , the bandwidth usage ratio is

$$
\frac{640}{(128 \times 5 \times 500) \times 2.2 \times(1-0.0015625)}=91.1 \% .
$$

As another example, with MaxSNR, when $n_{g}$ equals 32 , the bandwidth usage ratio is

$$
\frac{640}{(128 \times 5 \times 500) \times 2.65 \times(1-0.05)}=79.4 \% .
$$

Note that these theoretical values are very close to the simulation results values presented in Figure 6. This confirms the correctness of the simulation results.

4.4. Buffer Occupancy, Packet Delay, and Jitter. Figures 7(a), 7(b), and 7(c), respectively, show the mean buffer occupancy, the mean packet delay, and the mean packet jitter (RR performances are not presented in Figures 7(a) and 7(c) since its performances are not in the same order of magnitude. RR is not competitive here with the opportunistic schedulers). Section 4.3 points out the three phenomena that yield a higher system capacity when the bandwidth granularity increases. This gain in system capacity has a direct impact on the system performances in terms of mean buffer occupancy, delay, and jitter as long as the overhead impact is not too important. As expected, when $n_{g}$ increases until the 


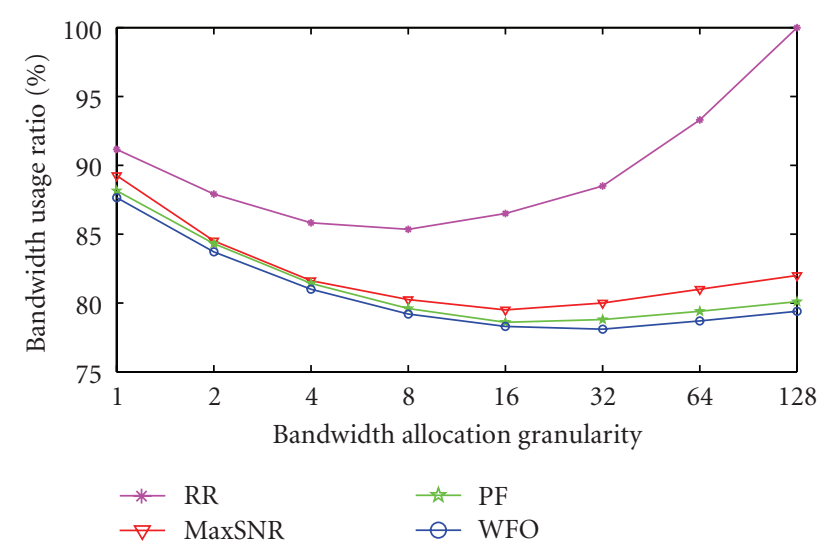

FIgURE 6: System capacity analysis.

optimum value cited in previous subsection, these three performance parameters (buffer occupancy, delay, jitter) improve for each scheduler thanks to a better spectral management since the gain due to supplementary frequency diversity is greater than the generated overhead. Additionally, it is important to note that the QoS improvement is very quick when the bandwidth granularity is incremented from 1 to 2. Afterwards, the gain is limited for all opportunistic schedulers. This shows that it is very advantageous to offer at least a few bandwidth granularity for improving these performance metrics.

4.5. Fairness and Mobile Satisfaction. Fairness is the most difficult objective to reach. It consists in ensuring the same QoS to all mobiles, whatever their position. In Figure 8, we examine the mobile dissatisfaction in terms of delay, discriminating with respect to the mobile position. We consider a mobile is dissatisfied when, at the end of its connection, its delay constraint is not met, that is, its experienced PDOR is greater than its PDOR target. So, the mobile dissatisfaction ratio is the percentage of mobile connections that do not satisfy the PDOR target. It is interesting to note that the four tested schedulers have not the least the same behavior when the amount of bandwidth granularity available in the system increases.

Classical RR yields bad results. Indeed, since multiuser diversity is not exploited, the overall spectral efficiency is small and system throughput is low. Consequently, the delay targets are widely exceeded and mobiles are dissatisfied. Naturally the spectral efficiency improves as the bandwidth granularity increases (Figure 5(a)). When the value of $n_{g}$ is maximum, all schedulers succeed to transmit more bits on a subcarrier than when $n_{g}$ is lower. However, the price to pay for a bandwidth granularity increase is a high overhead ratio growth. A bandwidth granularity increase is not beneficial to all the schedulers. For example, when $n_{g}$ equals 128 , only $80 \%$ of the capacity stays available for managing the useful data traffic (Figure 5(b)). In the case of RR, the gain provided by increasing $n_{g}$ from 64 to 128 is negligible (Figure 5(a)) compared to the high overhead ratio increase (Figure 5(b)). This induces a system overload. As shown in Figure 6, 100\% of the system RUs are used but much more are required. Indeed, the number of packets waiting for transmission builds up in the system, the packet delay values explode (Figure 7(b)), and, consequently, the users cannot be satisfied since their QoS requirements are not fulfilled (Figure 8). In conclusion, the results regarding mobile satisfaction (Figure 8 ) are highly related to the bandwidth usage ratio results (Figure 6), themselves depending on the joint results of spectral efficiency and overhead (Figures 5(a) and 5(b)).

Highly unfair, MaxSNR fully satisfies the required QoS of close mobiles at the expense of the satisfaction of far mobiles. Unnecessary priorities are given to close mobiles who easily respect their QoS constraints while more attention should be given to the farther. This inadequate priority management dramatically increases the global mobile dissatisfaction. Focusing on results of Figure 8(c), we observe that the mobile satisfaction slowly increases at first then decreases with the addition of bandwidth granularity. Indeed, opportunistic schedulers intrinsically need diversity. The higher the bandwidth granularity in the system is the more the MaxSNR scheduler takes advantage of it. Improving the spectral efficiency, the mobile satisfaction is directly impacted as far as the cost in terms of overhead is not too large. PF is subject to the same tendency and is even more reactive.

In contrast, with WFO, the easy satisfaction of close mobiles (with better spectral efficiency) offers a degree of freedom which is exploited in order to help the farther ones. WFO dynamically adapts the priorities as a function of the mobile position. This results in a fair allocation of the RUs providing the same level of QoS for each group of mobiles whatever their respective position. However, WFO needs more bandwidth granularity than the other schedulers for well developing its abilities. If we focus on the global mobile dissatisfaction (Figure 8(c)), with low bandwidth granularity ( $n_{g}$ equals 1$)$, all the other tested schedulers offer a greater number of satisfied mobiles. But, this supposes the sacrifice of the far mobiles in order to guarantee the QoS constraints of close mobiles. When $n_{g}$ equals 1 , WFO has not the means to an end. However, in contrast with the other schemes, as soon as $n_{g}$ increases, WFO takes benefit of the supplementary diversity and very quickly improves the QoS provided to all mobiles, widely outperforming the other schedulers.

\section{Discussion}

It appears that the performance of the considered schedulers in terms of system capacity, delay, jitter, fairness, QoS support, and so forth is widely different. As underlined in Figures 4, 6, 7, and 8 each scheduler is bandwidth granularity dependent and does not have the same behavior as a function of the bandwidth granularity variation. The respective performance of the studied schedulers also changes with the bandwidth granularity context. Additionally, Section 4 showed that it differs depending on the studied metrics too. For instance, PF outperforms WFO in terms of mobile satisfaction when $n_{g}$ equals 1 but the situation is different if we focus on the mean delay, jitter, or spectral efficiency. Moreover, regarding mobile satisfaction, when $n_{g}$ increases 


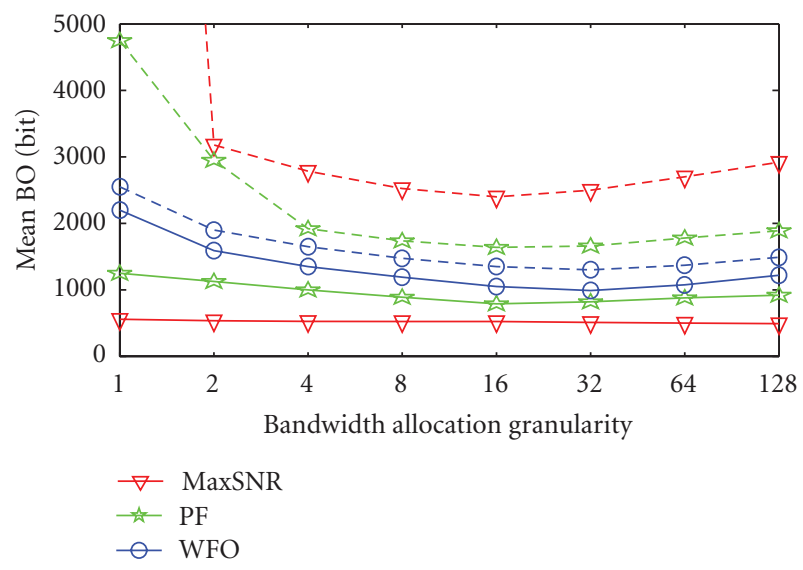

(a) Mean buffer occupancy for close mobiles (solid lines) and far mobiles (dashed lines)

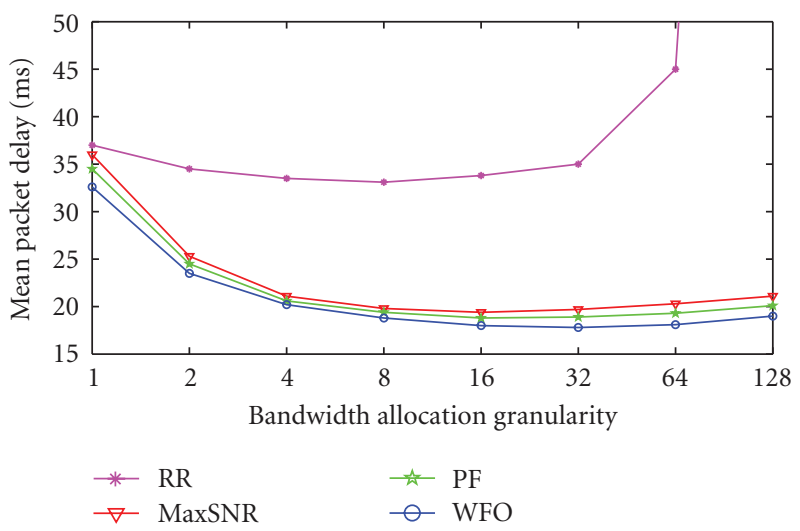

(b) Mean packet delay

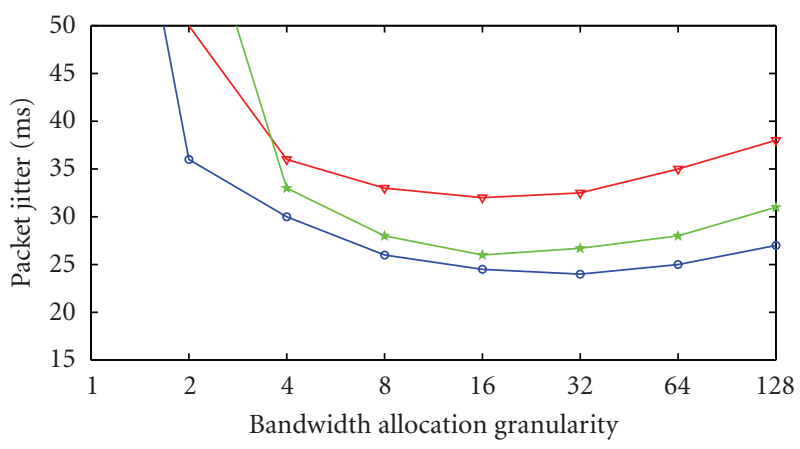

(c) Mean packet jitter

Figure 7: Buffer occupancy, delay, and jitter.

and reaches 4, WFO outperforms PF. According to the results presented in this paper, it seems really important to have a precise knowledge of the available bandwidth granularity in the system for choosing the scheduler. With a low bandwidth granularity, the opportunistic schedulers do not provide a large gain compared to the classical Round Robin scheduling which is much less complex. On the contrary, the higher the bandwidth granularity, the more interesting is the use of evolved schedulers. Indeed, a low bandwidth granularity highly degrades the advantages provided by each opportunistic scheduler. If we focus on the spectral efficiency, Figure 5(a) shows that for a value of $n_{g}$ running from 128 to 1 , the mean number of bits transmitted per allocated RU, respectively, decreases from 3.37 to 2.2 which corresponds to a fall by one-third.

In addition, this study demonstrates that, whatever the considered scheduling, full sharing of the bandwidth in many groups of subcarriers is not profitable for the system. Indeed, for each scheduler a tradeoff exists for a bandwidth granularity $n_{g}$ ranging between 8 and 32 . Higher $n_{g}$ values provide a too large overhead.

\section{Conclusion}

In this paper, we have compared the major resource allocation strategies for OFDM wireless networks. In addition, we have isolated the bandwidth granularity factor in order to study its influence on the inherent multiplexing gain offered by the OFDM schedulers. Comparing them for different bandwidth granularity values, this study has underlined that each scheduler is highly bandwidth granularity dependent and that performances are strongly related to this factor. When the bandwidth granularity increases, the performance variations depend on the studied performance metrics and the type of scheduler. Each scheduler have a limited application range with respect to the focused metrics. For instance, regarding delay and jitter, WFO seems to offer the best solution whatever the bandwidth granularity available while in terms of mobile satisfaction it outperforms PF and MaxSNR only in a context where bandwidth granularity is reasonable. Moreover jointly focusing on all performance metrics, the RR scheduler with low complexity is not too bad in a low bandwidth granularity context while MaxSNR, 


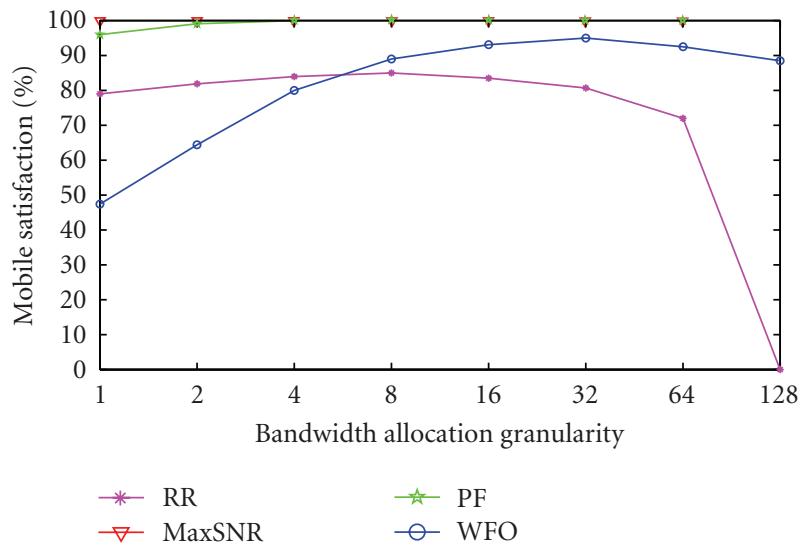

(a) Close mobiles satisfaction

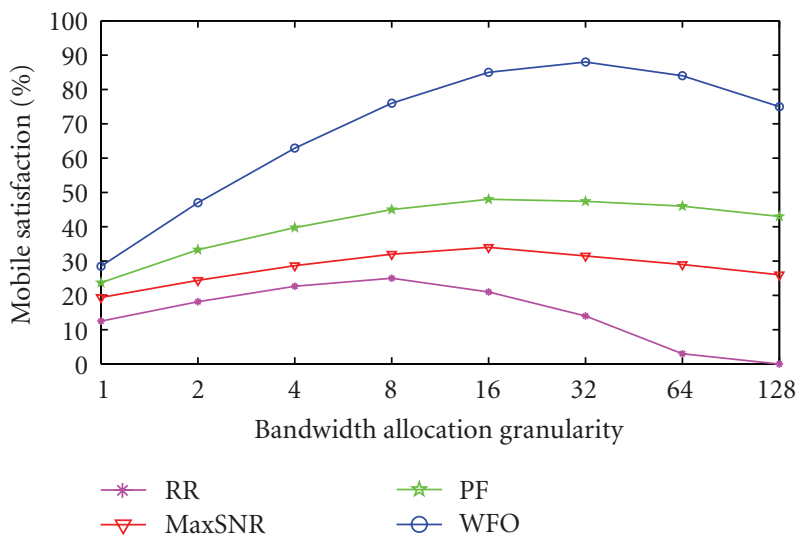

(b) Far mobiles satisfaction

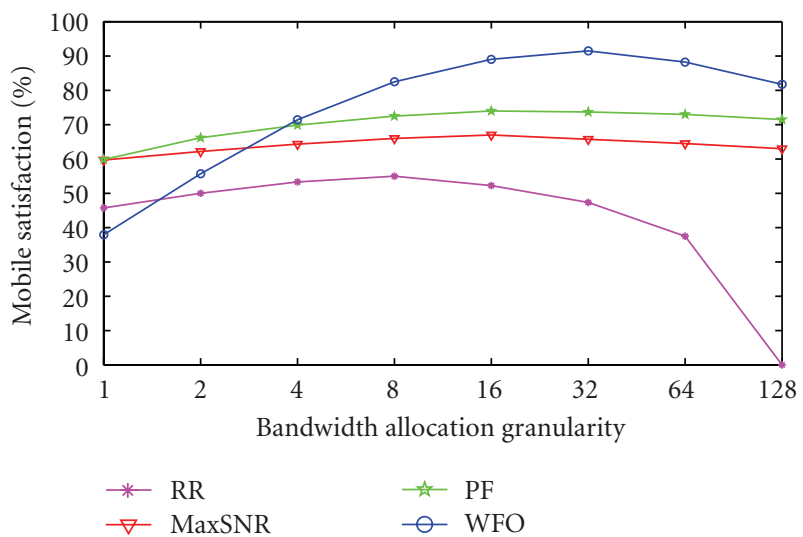

(c) Global mobile satisfaction

FIgURe 8: Analysis of the mobile satisfaction.

PF, and WFO are mainly designed for operating in highly frequency diversified systems.

\section{References}

[1] J. G. Proakis, Digital Communications, McGraw-Hill, New York, NY, USA, 3rd edition.

[2] R. Steele and L. Hanzo, Mobile Communications, IEEE Computer Society Press, 2000.

[3] A. Goldsmith, Wireless Communications, Cambridge University Press, 2005.

[4] J. D. Parsons, The Mobile Radio Propagation Channel, Wiley, 1992.

[5] R. Knopp and P. A. Humblet, "Information capacity and power control in single-cell multiuser communications," in Proceedings of the IEEE International Conference on Communications, vol. 1, pp. 331-335, June 1995.

[6] C. Y. Wong, R. S. Cheng, K. B. Letaief, and R. D. Murch, "Multiuser OFDM with adaptive subcarrier, bit, and power allocation," IEEE Journal on Selected Areas in Communications, vol. 17, no. 10, pp. 1747-1758, 1999.

[7] P. Viswanath, D. N. C. Tse, and R. Laroia, "Opportunistic beamforming using dumb antennas," IEEE Transactions on Information Theory, vol. 48, no. 6, pp. 1277-1294, 2002.
[8] H. Kim, K. Kim, Y. Han, and S. Yun, "A proportional fair scheduling for multicarrier transmission systems," in Proceedings of the IEEE 60th Vehicular Technology Conference (VTC '04), vol. 2, pp. 409-413, September 2004.

[9] C. Gueguen and S. Baey, "Scheduling in OFDM wireless networks without tradeoff between fairness and throughput," in Proceedings of the 68th Semi-Annual IEEE Vehicular Technology (VTC '08), September 2008.

[10] H. Zhou, D. Yang, W. Qi, and M. Ma, "On performance of mutiuser diversity in SISO and MIMO wireless communication," in Proceedings of the 14th IEEE International Symposium on Personal, Indoor and Mobile Radio Communications (PIMRC '03), vol. 3, pp. 2872-2876, September 2003.

[11] M. Sánchez-Fernández, M. Luz Pablo-González, and A. Lozano, "Exploiting multiuser diversity through uplink scheduling," in Proceedings of the IEEE 61st Vehicular Technology Conference (VTC '05), vol. 3, pp. 1918-1920, June 2005.

[12] J. G. Choi and S. Bahk, "Cell-throughput analysis of the proportional fair scheduler in the single-cell environment," IEEE Transactions on Vehicular Technology, vol. 56, pp. 766$7778,2007$.

[13] K. Kansanen and R. R. Müller, "Multiuser diversity in channels with limited scatterers," in Proceedings of the IEEE 19th International Symposium on Personal, Indoor and Mobile Radio Communications (PIMRC '08), pp. 1-5, September 2008. 
[14] T. W. Ban, W. Choi, B. C. Jung, and D. K. Sung, "Multi-user diversity in a spectrum sharing system," IEEE Transactions on Wireless Communications, vol. 8, no. 1, pp. 102-106, 2009.

[15] X. Zhang, W. Wang, and X. Ji, "Multiuser diversity in multiuser two-hop cooperative relay wireless networks: system model and performance analysis," IEEE Transactions on Vehicular Technology, vol. 58, no. 2, pp. 1031-1036, 2009.

[16] A. Tajer and X. Wang, "Multiuser diversity gain in cognitive networks with distributed spectrum access," in Proceedings of the 43rd Annual Conference on Information Sciences and Systems (CISS '09), pp. 135-140, March 2009.

[17] IEEEStd802.16-2004, "IEEE standard for local and metropolitan area networks, part 16: air interface for fixed broadband wireless access systems," IEEE Std 802. 16-2004, 2004.

[18] C. Hoymann, "Analysis and performance evaluation of the OFDM-based metropolitan area network IEEE 802.16," Computer Networks, vol. 49, no. 3, pp. 341-363, 2005.

[19] C. B. Ribeiro, K. Hugl, M. Lampinen, and M. Kuusela, "Performance of linear multi-user MIMO precoding in LTE system," in Proceedings of the 3rd International Symposium on Wireless Pervasive Computing (ISWPC '08), pp. 410-414, May 2008.

[20] M. Andrews, K. Kumaran, K. Ramanan, A. Stolyar, P. Whiting, and R. Vijayakumar, "Providing quality of service over a shared wireless link," IEEE Communications Magazine, vol. 39, no. 2, pp. 150-153, 2001.

[21] J.-J. Van De Beek, P. O. Börjesson, M.-L. Boucheret et al., "Time and frequency synchronization scheme for multiuser OFDM," IEEE Journal on Selected Areas in Communications, vol. 17, no. 11, pp. 1900-1914, 1999.

[22] X. Wang and W. Xiang, "An OFDM-TDMA/SA MAC protocol with QoS constraints for broadband wireless LANs," Wireless Networks, vol. 12, no. 2, pp. 159-170, 2006.

[23] C. Gueguen and S. Baey, "Compensated proportional fair scheduling in multiuser OFDM wireless networks," in Proceedings of the 4th IEEE International Conference on Wireless and Mobile Computing, Networking and Communication (WiMob '08), pp. 119-125, October 2008.

[24] H. Kim, K. Kim, Y. Han, and J. Lee, "An efficient scheduling algorithm for QOS in wireless packet data transmission," in Proceedings of the 13th IEEE International Symposium on Personal, Indoor and Mobile Radio Communications (PIMRC' 02), vol. 5, pp. 2244-2248, September 2002.

[25] A. Wang, L. Xiao, S. Zhou, X. Xu, and Y. Yao, "Dynamic resource management in the fourth generation wireless systems," in Proceedings of the International Conference on Communication Technology (ICCT '03), vol. 2, pp. 1095-1098, April 2003.

[26] P. Svedman, S. K. Wilson, and B. Ottersten, "A QoS-aware proportional fair scheduler for opportunistic OFDM," in Proceedings of the IEEE 60th Vehicular Technology Conference (VTC '04), vol. 1, pp. 558-562, September 2004.

[27] J. M. Holtzman, "Asymptotic analysis of proportional fair algorithm," in Proceedings of the 12th International Symposium on Personal, Indoor and Mobile Radio Communications (PIMRC '01), vol. 2, pp. F33-F37, October 2001.

[28] C. Gueguen and S. Baey, "An efficient and fair scheduling scheme for multiuser OFDM wireless networks," in Proceedings of the IEEE Wireless Communications and Networking Conference (WCNC '08), pp. 1610-1615, April 2008.

[29] S. Baey, "Modeling MPEG4 video traffic based on a customization of the DBMAP," in Proceedings of the International Symposium on Performance Evaluation of Computer and Telecommunication Systems (SPECTS '04), July 2004.
[30] P. Brady, "A model for generating on-off speech patterns in two-way conversation," Bell System Technical Journal, vol. 48, 1969. 

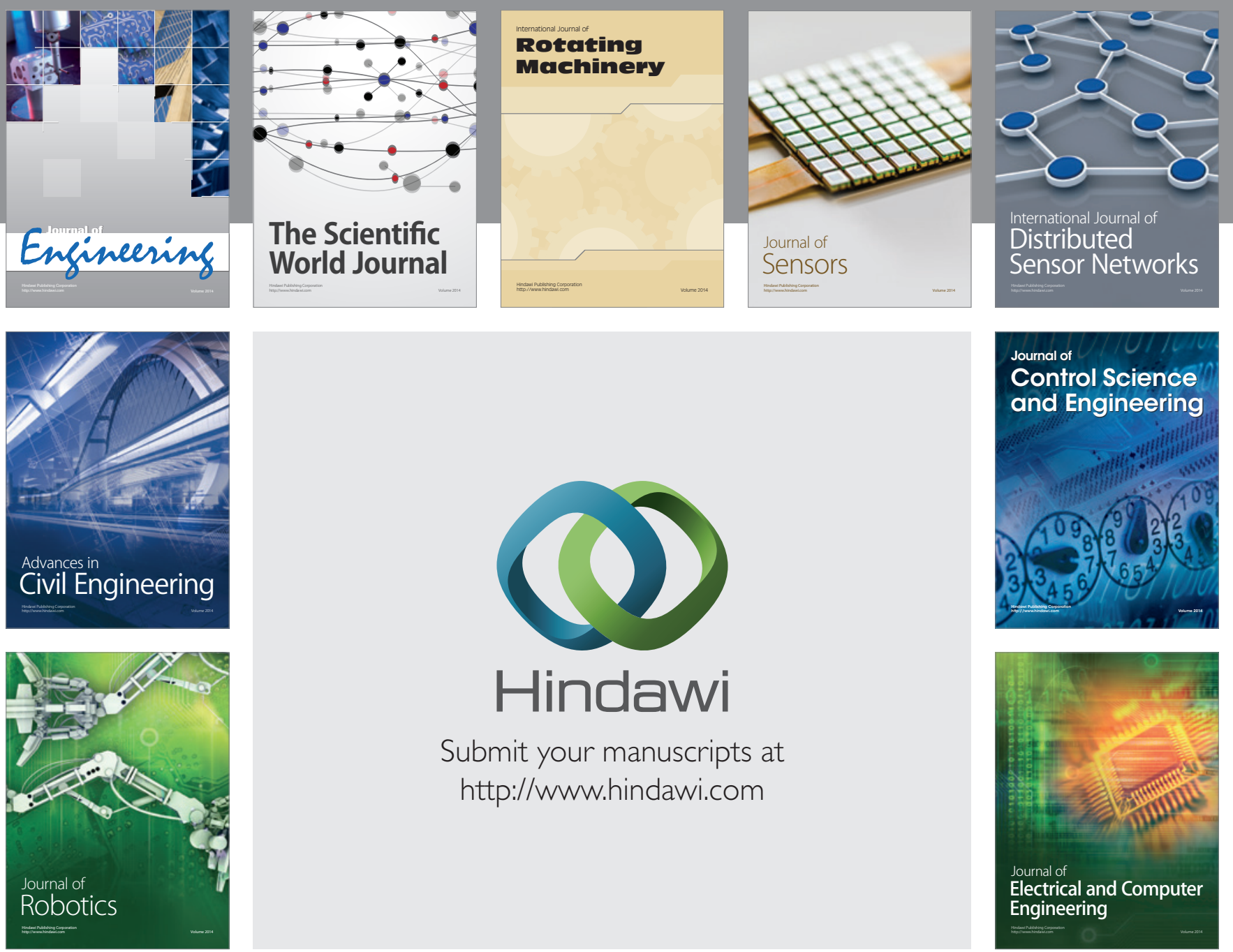

Submit your manuscripts at

http://www.hindawi.com
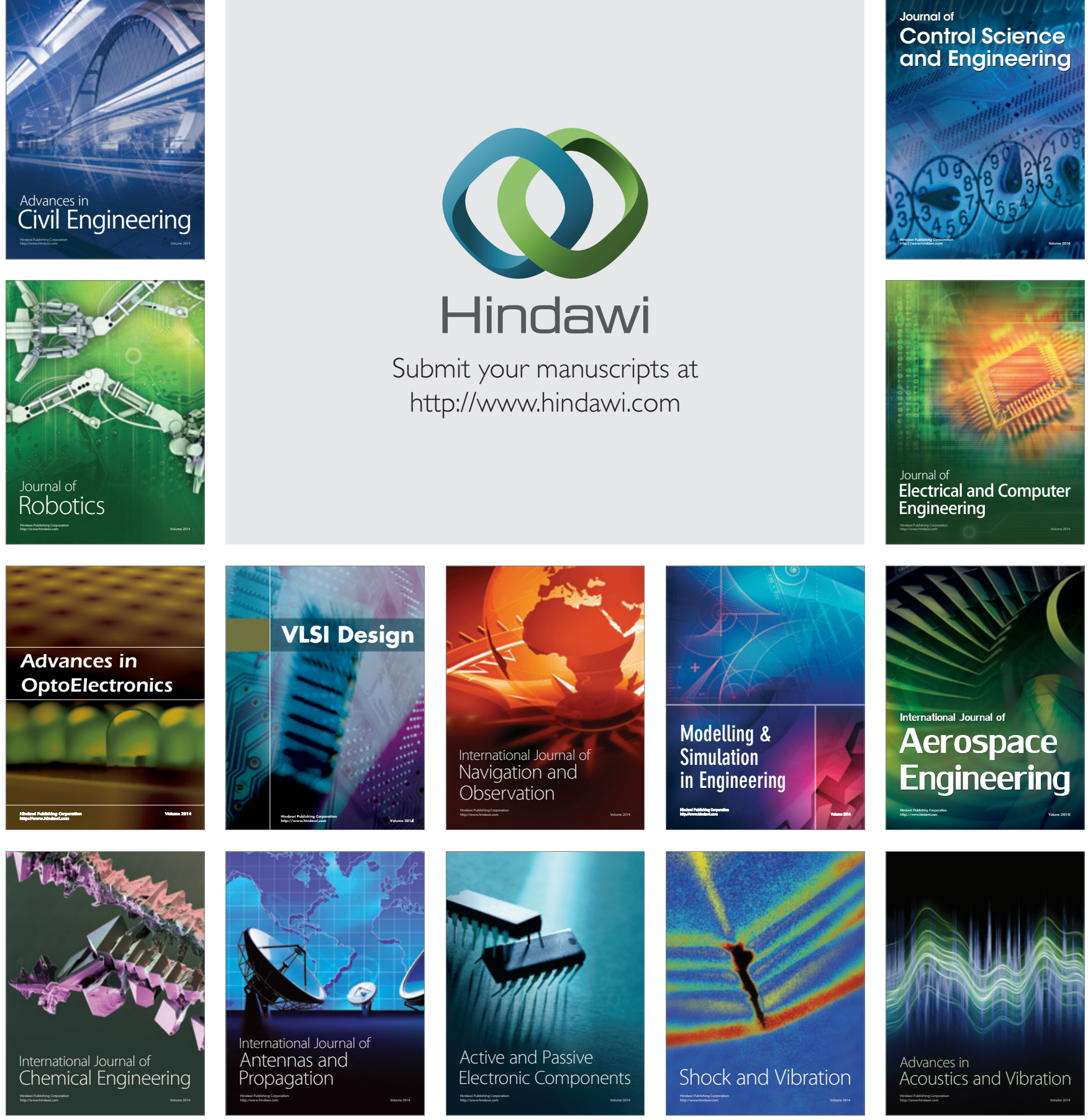